\title{
Rapid HLA-DPB typing using enzymatically amplified DNA and nonradioactive sequence-specific oligonucleotide probes
}

\author{
Teodorica L. Bugawan, Ann B. Begovich, and Henry A. Erlich
}

We wish to clarify some of the issues related to cloning polymerase chain raction (PCR) products amplified with the HLA-DPB1 primers UG19 and UG21 described in Bugawan and co-workers (1990). These primers were designed with restriction sites added to their $5^{\prime}$ ends in order to facilitate cloning. UG19 (the left primer) has one strand of the Pst I site and UG21 (the right primer) has one strand of the Bam HI site. However, there is an internal $P_{s t}$ I site (codons 89 and 90 ) within the DPB1 second exon amplified by these primers so that complete Bam HI and Pst I digestion creates a 261-bp Pst I fragment. Consequently, cloning a Bam HI-Pst I digested PCR product into a Bam HI-Pst I cut vector results in very low cloning efficiency. One solution to this problem is to clone $P s t$ I digested PCR products amplified with UG19 and UG21 into a Pst I digested vector that has been treated with bacterial alkaline phosphatase to minimize self-ligation of the vector. Alternatively, the PCR products can be blunt-end cloned or alternative DPB1 primers (e.g., DB01, CAGGGATCCGCAGAGAATTAC, described in Bugawan and co-workers (1988); a "left" primer with a Bam $\mathrm{HI}$ site) can be used. We regret any inconvenience experienced in cloning DPB1 sequences amplified with these primers and hope this note clarifies the situation.

\section{Reference}

Bugawan, T. L., Horn, G. T., Long, C. M., Mickelson, E., Hansen, J. A., Ferrara, G. B., Angelini, G., and Erlich, H. A.: Analysis of HLA-DP allelic sequence polymorphism using the in vitro enzymatic DNA amplification of DP $\alpha$ and DP $\beta$ loci. J Immunol 141: 4024-4030, 1988 